\title{
Research on the Architecture of Internet of Things and Related Technologies
}

\author{
Xia Wei \\ Xi'an International University, Xi’an, Shaanxi, 710077
}

Keywords: Internet of Things; Architecture; Technology

\begin{abstract}
IOT is essentially a physical structure, based on the network, and IOT is undoubtedly an important product of the era of social science and technology. IOT technology has a (http://www.likenews.us/) widespread concern, according to the relevant data that Google search volume of "IOT" in 2016 is up to 100 million, showing that the attention, so IOT is most popular. It is necessary to carry out an in-depth study and analysis of IOT under the background of this kind of environment. This paper analyzes the background and connotation of IOT, and then studies the IOT architecture. At last, this paper will elaborate the actual promotion mode of IOT technology level, with a view to providing theoretical basis for the follow-up study on IOT.
\end{abstract}

\section{Introduction}

Into the new era, "IOT" in the context of the rapid development of information technology came into being, we can say "IOT" will be an important future direction of science and technology, and "IOT" technology can really affect the social life and production aspects, The country to modernize the development of the road one of the key means of application, and for the "IOT" in terms of its architecture in the comprehensive information and data perception and intelligent processing and the corresponding mass storage analysis, you can virtual network and the corresponding real life To establish a close relationship in this regard is conducive to the transformation of the national industrial and agricultural production mode, on the other hand is also conducive to enhancing the effective utilization of resources in real terms.

\section{The "IOT" Development and Connotation}

"IOT" was first produced in 1999, is made by the United States network meeting specifically, when few people to be concerned about it until 2008 when the "IOT" to achieve the initial popularity and began to be national attention, and "IOT "IOT" technology has been applied in the field of pharmaceutical and logistics and corresponding retailing, and the application effect is more gratifying, such as the current high-speed (high-speed) and high-speed The new charge system and the mobile wallet are based on the "IOT" design, according to the relevant research scholars predict the next five to ten years between mankind will enter the smart era, which also indicates that the " IOT "will achieve a full range of applications and widespread popularity [1].

The definition of "IOT" in the current international alliance is based on the relationship between people and objects and between people and people. The "IOT" is regarded as an Internet based on computer Internet Based on the information transfer between the collection and processing of the implementation process; and our government for the "IOT" is defined as relying on the sensing device to achieve the interaction of objects and the connection object and the corresponding monitoring object information collection and identification of transmission , And the establishment of the Internet based on the formation of a huge network, the ultimate goal of development is to effectively connect people and things and objects, to facilitate information control and management [2]. 


\section{The "IOT" System Architecture}

The "IOT" is evolving, and from the "IOT" in essence, it is an inter-related items between the system structure, the user as the core of the system, relying on embedded in the World Wide Web to achieve good user information management, Which is based on wireless communication. The important technology of the design is based on the autonomy, and it can be divided into two parts: "end node" to "autonomous element" and then "intermediate node", then "intermediate node" Transmission to the "autonomy", and finally to the "end" to achieve the completion of network control-related tasks, and evolutionary communication system is also one of the key features. For the "IOT" its architecture includes the management level and the knowledge level and the corresponding control level, the data level, in which the management level directly to the knowledge and control and the corresponding data three structure layer management. From the knowledge level in terms of its decision-makers to provide the relevant knowledge; from the control level in terms of its message with the transmission configuration and throughput optimization and other functions; and from the data level, it also contains three small First, from the data sensing layer, the structure layer belongs to the "IOT" infrastructure, occupies an important position in the "IOT", can be in the information data to the data layer and the data transmission layer and the corresponding application layer, After the actual acquisition of short-distance information transmission, mainly depends on wireless transmission and cable transmission technology to achieve for the background processing data and laid a solid foundation. Secondly, from the data transmission layer, "IOT" appears itself shoulder the important function of good data transmission responsibility, the layer is built on the basis of the perception layer, the data collected on the previous layer to achieve effective long-distance transmission, and the transmission Layer has a high demand for Internet use, so a large extent, the actual Internet technology affects the effectiveness of the data transmission layer. Finally from the application layer, "IOT" ultimate development is reflected in the application layer, the application layer is built on the basis of the two layers, through the analysis of data processing to achieve reasonable control, more convenient application management to achieve human-computer interaction development goals, The general application layer is divided into the terminal application layer and the program layer, which makes the "IOT" achieve rapid development [3].

\section{The Analysis of "IOT" Related Technologies}

Network sharing is one of the "IOT" technologies, which are based on the Internet, and can be used to transmit information in the Internet. They can also be applied in different regional environments and show strong flexibility in network form. , Such as transportation and construction in the application of both, then the two aspects of the information will be able to achieve a good transfer to facilitate the construction of transport-related information in the management of grasp, and traffic construction can also be timely access to transportation information, Information sharing, and then for many users to be efficient and convenient network system, can now be said that "IOT" has been constantly overcome a single network development model to the road to the development of network sharing on the road.

Sensor sensing is also one of the "IOT" technologies. It is also a key technology in the current "IOT" system. It can be applied to the actual collection of data information, and can be used in microelectronics related technology to analyze the voice information, The temperature information and so on. It is a kind of processing tool in essence. It can make the information household realize the convenient and convenient access, and provide more accurate and scientific original data [4].

"IOT" technology In addition to the above two technologies, the Bluetooth is one of the technology is a new type of wireless sensor technology, but can not act on the distance, but only suitable for short distance between the good wireless Sensing, enabling high-frequency communications and multi-address communications, in addition to the technology can also be mobile devices and the corresponding computer information between the larger to simplify, with a 
wider range of transmission and more convenient delivery, now in various fields Industry to achieve effective application [5].

"IOT" technology also includes data processing, and the data is handled well in order to achieve the goal of man-machine dialogue, the technology also belongs to the "IOT" key technology, through the effective collection of data, and then intelligent research and analysis, and Will deal with the data after relying on a simple form of presentation, for the majority of users with a more convenient use experience [6].

RFID is one of the "IOT" technology, which can facilitate the exchange of a variety of data information, which is mainly composed of read and write devices and the antenna and the corresponding electronic tags in three parts, the implementation of close collaboration between the three data Information is a good complete reserve, in which the electronic tag itself has a storage function and the corresponding read and write devices with read data and write data the actual function of the antenna is used for signal reception and the corresponding signal transmission; its inherent advantage is reflected in the High level of automation and strong adaptability of the actual network environment and the corresponding rapid identification of information, it can be said that the technology, whether in the present or future have broad prospects for development [7].

ZICBEE, like RFID, is also one of the "IOT" technologies, which are in between Bluetooth and wireless tag technology, mainly in the process of transmission of data to be identified and the corresponding grouping frequency processing, the inherent technology Advantages in many areas, such as strong flexibility and low consumption and high reliability, strong security and large network capacity, the current technology in a number of industries to achieve the field of application, but need Improved the technology can only be applied to a small range of information transmission, but also need to be expanded in the scope of the role [8].

\section{The Analysis of "IOT" to Enhance the Level of Technology}

The first is that the government needs to give policy support to the "IOT" industry, and it can establish the "IOT" demonstration base, which can promote the "IOT" and other industries IOT "information standards, such as the development of interface standards and the corresponding implementation of standards and research and development standards, etc .; the other is the" IOT "to improve the basic equipment to improve the" IOT "to improve the standard, IOT "to enhance the actual development of information technology to build a unified platform for public information, the" IOT "architecture to further improve and strengthen the sharing of good information resources; the third is to develop" IOT " Talents and talents are the fundamental strength of "IOT", which requires enterprises and universities to attach importance to "IOT". Enterprises can provide "IOT" technical training to existing employees, and can also recruit a large number of "IOT" technical personnel, "IOT" is needed for higher education, and "IOT" is more powerful, and IOT innovation and advancement of the times can be realized by relying on these talents.

\section{Conclusion}

In summary, the emergence and development of "IOT" is not only the objective requirement of national progress, but also the inevitable demand of science and technology to lead the development of society. At present, many scholars need to carry out in-depth research on "IOT", from "IOT" IOT "network sharing technology, or Bluetooth and data processing technology can be applied in urban traffic and medical and the corresponding water conservancy. In this paper, the" IOT "technology is widely used, And so on, which undoubtedly play a vital role in urban development, and the government also need to be based on the "IOT" to be more policy help, and this paper, "IOT" technology and architecture as the core In the follow-up "IOT" optimize the development of their own a modest contribution. 


\section{References}

[1] Chen Haiming, Cui Li, Xie Kaibin. Comparison of IOT Architecture and Implementation Methods [J] .Journal of Computer, 2013, 01: 168-188.

[2] Sun Wei. Study on the structure and key technology of sensing mines based on Internet of Things [J]. Coal Technology, 2015, 06: 250-252.

[3] Li Yu, Lu Tingjie. Study on the Development Strategy of Internet of Things Industry Based on Industry Chain Perspective [J]. Journal of Beijing University of Posts and Telecom, 2012,04: 46-51.

[4] Sun Jie, Xu Hong. Internet of Things architecture and related software system code automation [J]. Microcomputer Information, 2012, 09: 329-331 +361.

[5] FAN Fan.Study on application and development of networking technology and architecture of port group in Guangxi Beibu Gulf [J]., 2011,10: 58-61.

[6] Zhu Hongbo, Yang Longxiang, Jinshi, Zhang Dengyin, Cheng Chonghu, Zhu Qi, Guo Yong'an. Cooperative Innovation System and Intelligent Service Industry of Internet of Things [J] .Journal of Nanjing University of Posts and Telecommunications, 2014,01: 1 -9.

[7] Zhu Xiaojing, Gu Donghu. Mobile Device Interaction Architecture Based on Internet of Things [J]. Information Technology and Informatization, 2014, 05: 131-132 + 161.

[8] Zhang Li, Yang Huaizhou. Research on Key Technologies of QoS-driven Service Composition in Internet of Things [J]. Information Technology and Informatization, 2016, 09: 60-65.

[9] Mi Yongwei, Wu Ruichang, Li Yiyong, Zhang Kai, Wang Wei, Li Tao. Medical equipment management system architecture and key technologies based on Internet of Things [J]. Medical Equipment, 2016, 03: 33-36. 